\title{
El Escenario de la Intervención Comunitaria
}

\author{
The scenary of Community Intervention
}

Antonio Ismael Lapalma*

\section{Resumen}

Este trabajo presenta y analiza los componentes para la construcción de un "escenario para la intervención comunitaria" que permita contextualizar el rol del psicólogo comunitario. A partir de la interrelación de tres aspectos: a) necesidades sociales; b) Organización; y c) el medio ambiente, y de su vinculación con los procesos de participación social, se construyen alternativas de cooperación, alianzas, o confrontación entre la diversidad de actores sociales presentes. Se exponen cuatro racionalidades básicas existentes en el campo de la intervención, tales como, la política, la técnica, la burocrática y de la población. Estos aspectos, examinados en un contexto histórico social determinado, pueden ser utilizados como instrumento de diagnóstico comunitario, de planificación estratégica y de evaluación, desde una perspectiva participativa.

Palabras Claves: Intervención comunitaria, Psicología comunitaria, metodología de interventión.

\section{Abstract}

This proyect present and analyses the elements for the construction of a "Scenario of Community Intervention" that enable to place the roles of the community psychologist into context.

Cooperation alternative, alliances or confrontatión between social actors are built as from the interrelation between the following aspects: a) social needs, b) social organization and c) environment and the linking of the processes of social participation.

Four basic existing rationalities are shown in the intervention fiel such, politicals, techinical, boreaucratic and the one related to the people. These aspect, examined in a determined social an historic context can be used as a tool for community diagnosis, strategic planning and assessment, fron a participating perspective.

Key words: Community intervencion, community psychology, intervention methodology

\footnotetext{
* Facultad de Psicología. Universidad de Buenos Aires. República Argentina e-mail: lapalma@house.com.ar
} 


\section{Introducción}

Desde el punto de vista de la psicología comunitaria la intervención social comunitaria hace referencia a procesos intencionales de cambio, mediante mecanismos participativos tendientes al desarrollo de recursos de la población, al desarrollo de organizaciones comunitarias autónomas, a la modificación de las representaciones de su rol en la sociedad y sobre el valor de sus propias acciones para ser activo en la modificación de las condiciones que las marginan y excluyen (Chinkes, Lapalma, Niscemboin, 1995).

Se desarrollan en un escenario social que el psicólogo comunitario debe construir para la comprensión de las «multifacéticas condiciones en las cuales se expresan las conductas en diversas condiciones ambientales» (Werstergaard, Kelly, 1992:36) y que permita la contextualización de las intervenciones que son parte de «un contexto social social multideterminado, multiestructurado y de múltiples niveles» (Werstergaard, Kelly, op.cit: 41).

La comprensión de la diversidad de los aspectos que constituyen un escenario social comunitario facilita el diagnóstico del mismo, el análisis de sus contenidos y el establecimiento de cursos de acción alternativos que son implementados mediante la asociación voluntaria entre la población y equipos técnicos, a través de procedimientos grupales que amplían la percepción crítica de la realidad.

Así "el papel de los expertos profesionales es entonces meter la reflexividad de los contextos en las preguntas de los expertos animadores (dirigentes), a través de las expresiones de los expertos vivenciales (bases) (Villasante, 1998,31). La reflexión de la realidad se instala en situaciones concretas y en su dimensión histórica.
Los siguientes aspectos han sido considerados para la construcción del escenario de la intervención comunitaria :

- 1) las necesidades sociales, 2) la diversidad de formas organizativas que surgen para modificarlas y 3) la interacción con otros actores sociales en un medio ambiente determinado.

- El reconocimiento de que estas relaciones se estructuran en un contexto histórico, político, económico y social determinado.

- La existencia de racionalidades específicas que representan a la diversidad de actores sociales. Entre ellas se destacan la política, la técnica, la burocrática y la correspondiente a la población.

Este escenario social complejo, turbulento, es el ámbito de procesos participativos en sus manifestaciones social, política, comunitaria y ciudadana, de articulación y de confrontación entre actores sociales.

\section{Desarrollo de sus Componentes}

\section{a. Las necesidades sociales}

Las necesidades sociales han sido estudiadas desde una variedad de perspectivas: como carencias y potencialidad (Max-Neef, 1993); como insatisfacción percibida (Montero, 1991); como un sistema triádico: deseo, necesidad, carencia (Heller, 1996); y como capacidades para funcionar (Amartya Sen, 1995).

Por la facilidad de operacionalizar y abordar las necesidades sociales en las distintas etapas del trabajo comunitario (involu-cramiento, diagnóstico, planificación participativa, organización comuni- 
taria y evaluación) se considera para este trabajo la siguiente clasificación de necesidades: subsistencia, identidad, libertad, ocio, creación, participación, entendimiento, afecto y protección (Max Neef, 1993) ${ }^{1}$. Las necesidades humanas conforman un sistema, en el cual no pueden establecerse criterios de jerarquías dado que se expresan a través de simultaneidades, comple-mentariedades y compensaciones (Max Neef, op.cit: 37). Las necesidades son carencias pero también potencialidades, en tanto recursos que movilizan a las personas.

A la clásica relación entre necesidad y recursos incluye la noción de satisfactor como la «modalidad dominante que una cultura o sociedad imprime a las necesidades (Max-Neef, op,cit: 44), siendo este el aspecto más dinámico de la propuesta.

Los satisfactores son formas organizativas, prácticas sociales, espacios, valores, modelos políticos, históricamente constituidos y su identificación permite el diseño de estrategias sinérgicas de intervención en el campo de las necesidades sociales. En el cuadro Nro. 1. Satisfactores. Caracterización, se muestra la diversidad de los mismos.
Otro de los aportes es el reconocimiento de que las necesidades, los satisfactores y los bienes tienen tiempos y ritmos diferentes. "Las necesidades cambian con la aceleración que corresponde a la evolución humana; los satisfactores tienen una doble trayectoria; por un lado se modifican al ritmo de la historia, por el otro se diversifican según la cultura. Los bienes tienen una triple trayectoria: 1) se modifican al ritmo de las coyunturas, 2) se diversifican dentro de la cultura y 3) lo hacen también de acuerdo con los estratos sociales. Estos aspectos cambian con velocidades diversas (Max Neef, op.cit.;49).

\section{b. Las organizaciones}

El interés por las organizaciones ha estado vinculado a organizaciones más complejas y antiguas como la administración, las empresas, las prisiones, las escuelas, hospitales, las organizaciones militares, los sindicatos. Y todas ellas poseen un grado de desarrollo organizacional e historia que le es específica. Se distinguen por una estructura diferenciada horizontal y vertical, lo cual ordena un sistema de roles asignados, y su tendencia es a la burocratización (Mayntz, 1967:27).

1 Esta propuesta, conocida como "Desarrollo a Escala Humana», considera al desarrollo social y las necesidades como componentes de una ecuación irreductible. (Max-Neef, 1993:33). Las ideas se originan en el Proyecto GPID: Goald, Process and Indicators of Development Proyect", iniciativa internacional y multiorganizacional que estudiaba alternativas de desarrollo al nuevo orden internacional. 


\section{Cuadro Nro. 1.}

Satisfactores. Caracterización según Max Neef

\begin{tabular}{|c|l|}
\hline Satisfactores & \multicolumn{1}{|c|}{ Características } \\
\hline Violadores o destructores & $\begin{array}{l}\text { A mediano plazo aniquilan la posibilidad de su } \\
\text { satisfacción y, por sus efectos colaterales, } \\
\text { impiden la satisfacción de otros, son impuestos. } \\
\text { Ej. Censura. }\end{array}$ \\
\hline Pseudo-satisfactores & $\begin{array}{l}\text { Producen una falsa sensación de satisfacción, } \\
\text { pueden aniquilar la necesidad que pretenden } \\
\text { satisfacer. Son inducidos. Ej. Sobreexplotación de } \\
\text { recursos ambientales. }\end{array}$ \\
\hline Inhibidores & $\begin{array}{l}\text { Sobresatisfacen, pero dificultan la posibilidad de } \\
\text { satisfacer otras necesidades. Están ritualizados. } \\
\text { Ej. Aula autoritaria. }\end{array}$ \\
\hline Sinérgicos & $\begin{array}{l}\text { Satisfacen a una y estimulan y contribuyen a la } \\
\text { satisfacción simultánea de otras. . Ej. Lactancia } \\
\text { materna. }\end{array}$ \\
\hline Singulares & $\begin{array}{l}\text { Apuntan a la satisfacción de una sola necesidad, } \\
\text { siendo neutros de otras. Ej. Planes alimentarios. }\end{array}$ \\
\hline
\end{tabular}

Para este autor las organizaciones más pequeñas, con poca interna, estructuradas por lazos familiares o de comunidad, de cercanía geográfica, donde el trabajo es una forma de vida, más que un desempeño de funciones delimitadas, no entran en la categoría de organizaciones.

Sin embargo en el ámbito comunitario se observa una diversidad de organizaciones, que tienen complejas formas de funcionamiento, que fueron reconocidas por numerosos autores tales como las estrategias de sobrevivencia (Bartomé, 1981) que funcionan como el conjunto de procedimientos, selección y utilización de recursos para la satisfacción de necesidades, o como redes de ayuda mutua (Aguirre, 1981), o los recursos que implica la pertenencia a una red de interacción social en el renovado concepto de capital social (Portes, 1999).

Katz y Kahn (1981) sistematizan la diversidad de modelos organizacionales que dan cuenta de estos procesos y señalan que en las organizaciones comunitarias pueda darse una combinatoria de estos tipos.

I. Organizaciones que poseen una estructura poco diferenciada, surgen por estímulo o influencia del medio, para resolver problemas comunes de sus integrantes. Las tareas se realizan basadas en la cooperación y solidaridad (todos hacen de todo). La estructura está basada en valores y expectativas compartidas y el principio de autoridad está centralizado en un liderazgo personalizado. 
II. Además de las necesidades comunes observadas en el párrafo anterior, en la estructura aparecen diferenciaciones internas, originadas por aspiraciones personales, por aprendizaje social o por capacidades individuales para resolver nuevas demandas del medio ambiente. Los objetivos, así como las reglas de funcionamiento, comienzan a ser explicitadas. La autoridad es una función diferenciada coexistiendo a veces con el liderazgo personalizado.

III. Presentan una mayor complejidad; sus objetivos y normas están escritos para todos los integrantes, la división del trabajo está basada en roles y funciones y la autoridad es un rol. Aparecen mecanismos de coacción para el cumplimiento de las normas y reglas.

Para estos autores las organizaciones, los movimientos comunitarios y los grupos de voluntarios se basan en los dos primeros mecanismos, más que en el tercero. En el cuadro Nro. 2 - Diversidad Organizacional, se señalan los aspectos considerados (objetivos, autoridad, división de trabajo y normas) y la posibilidad de múltiples combinaciones entre ellos.

Cuadro Nro. 2.

Diversidad organizacional

\begin{tabular}{|l|l|l|l|}
\hline \multicolumn{1}{|c|}{ Aspectos } & \multicolumn{1}{c|}{ I } & \multicolumn{1}{c|}{ II } & III \\
\hline Objetivos & Implícitos & Explícitos & Escritos \\
\hline Autoridad & Liderazgo & Según tarea & Función \\
\hline & personalizado & & \\
\hline División del Trabajo & $\begin{array}{l}\text { Cooperativo y } \\
\text { solidario }\end{array}$ & $\begin{array}{l}\text { Roles iniciales } \\
\text { y por tarea }\end{array}$ & $\begin{array}{l}\text { Roles } \\
\text { y funciones }\end{array}$ \\
\hline Normas & Afectivas & Explícitas & Escritas \\
\hline
\end{tabular}

La utilización y la combinación de estos aspectos son importantes para el diagnóstico de las organizaciones comunitarias, para evitar modelos normativos o ideales de las organizaciones. Merece especial atención este señalamiento dado que, en la década del 90 , surge una revalorización del rol de la sociedad civil bajo el nombre de «tercer sector» y de sus organizaciones de base, con el riesgo de que las organizaciones comunitarias queden atrapadas en las «miradas» de las de tipo III, como modelos de eficacia y eficiencia a los cuales arribar, excluyendo o minimizando de esa manera la capacidad organizativa y de movilización que poseen las que transitan por distintas combinatorias entre los tipos I y II.

La población, a través de esta diversidad de formas organizativas, interactúa entre sí e intenta satisfacer las necesidades sociales, en un espacio social y territorial correspondiente: el campo de la participación comunitaria. Esta heterogeneidad de organizaciones se inserta en un espacio histórico, social y económico específico, al cual denominaremos: medio ambiente. 


\section{c. El medio ambiente}

Hace referencia al espacio histórico, político, socio económico y cultural caracterizado por la existencia de actores sociales, ${ }^{2}$ a quienes referiremos como personas, grupos, instituciones cuyo comportamiento está orientado por sus intereses y ejercen mecanismos de influencia en su defensa.

El medio ambiente es un espacio de ejercicio de posiciones de poder. "El poder es una relación: no se puede desarrollar más que a partir del intercambio de los actores comprometidos en una determinada relación, pues en la medida en que toda relación entre dos partes supone intercambio y adaptación entre ambas, el poder está inseparablemente ligado a la negociación: es una relación de intercambio por lo tanto de negociación." (Croizier, Friedberg, 1990:56).

El medio ambiente es un espacio social y territorial conflictivo: los actores sociales se articulan, establecen mecanismos de cooperación, alianza, confrontan y negocian.

De una mirada más amplia entre la población, sus necesidades, las organizaciones y la trama multiorganizacional, surgen los procesos de participación social orientada a la gestión de intereses sociales (Sánchez, E., 1994) que incorpora a la política, a través de la representación por medio de estructuras partidarias, comunitarias (necesidades sentidas y legitimación) y ciudadanas (defensa de intereses personales, mediante organizaciones o aspectos jurídicos de carácter general).
Podemos sintetizar los siguientes aspectos básicos del medio ambiente, conflictividad, poder y posibilidades de ejercer influencia activa para modificarlo.

\section{d. El Contexto}

Los aspectos estudiados, las necesidades sociales, las organizaciones y el medio ambiente, los procesos participativos, las racionalidades existentes se hallan insertos y muchas de las veces determinados por un espacio histórico, económico y social, mucho más abarcador, que ejerce influencia y al que no es posible modificarlo. Lo denominaremos el contexto:

Cuando es posible su modificación, para su operacionalización, en el marco de este trabajo lo llamaremos medio ambiente. A modo de ejemplo: un programa de ajuste económico, para la mayoría de la población, es un determinante de sus condiciones de vida. Para los grupos económicos, que pueden imponer condiciones, ejercer su influencia en el programa, es su medio ambiente. Si la población, a través de la movilización, la protesta, por la construcción de movimientos sociales o a través de la organización política puede ejercer influencia y modificarlo, la situación de contexto se ha transformado en una situación del medio ambiente. Esta diferenciación es de capital importancia en los procesos de planificación estratégica.

Es posible señalar dos espacios-contextos: el primero, «externo», que podría estar representado por la "globalización", y que señala la adopción generalizada y triunfan-

2 "Sujetos individuales o colectivos que, en una situación dada, controlan o manejan recursos suficientes que les permiten ejercer algún grado de influencia sobre los elementos que conforman dicha situación. Pueden ser recursos económicos y financieros, la información, el conocimiento y las tecnologías, la capacidad de convocatoria y de movilización, la capacidad de posicionamiento, relacionamiento y articulación, la capacidad de gestión. 
te del «discurso neoliberal ${ }^{3}$ ", una transnacionalización y volatibilidad del capital, facilitado por la revolución tecnológica de las comunicaciones y la informática.

En el segundo de estos espacios cercanos aparece la reforma del Estado (achicamiento), los programas de ajuste estructural de la economía, la desregulación, la apertura económica las privatizaciones, la deuda externa y su influencia en el medio ambiente: desempleo, flexibilidad laboral, creciente pobreza y la exclusión social y los efectos psicosociales de la misma.

La exclusión social se refiere por un lado a la situación experimentada por los individuos (privación económica y aislamiento social) y por otro, a un proceso social, que aparece como la fragmentación social, los dualismos (pocos ricos muy ricos y muchos pobres muy pobres) y la ruptura de la cohesión social. (Figueroa, Altamirano, Sulmont, 1996).

Un grupo social se considera excluido cuando no se le permite participar de algunas relaciones del proceso social que considera valioso. Implica una reducción global del control que una sociedad ejerce sobre los riesgos sociales.

La exclusión abarca tres dimensiones: económica (cuando los sujetos no pueden o no cuentan con los medios para participar de los procesos productivos); política: referida al ordenamiento político institucional que regula el ejercicio del poder, establece nor- mas, deberes, garantiza derechos y la participación activa en lo que llamamos ciudadanía (educación, salud básica, seguridad social) y cultural, que corresponde a los códigos, valores y aspiraciones mediante los cuales las personas se comunican entre sí, interpretan la realidad y orientan su práctica, que se transmite a través de las relaciones primarias, la educación, la religión y los medios de comunicación.

\section{Acerca de las Racionalidades Existentes}

Observamos cuatro racionalidades básicas; la política, la técnica; la burocrática y de la de la población y que poseen: una cosmovisión del escenario de la intervención y de actores sociales presentes en él. Tienen diferentes lenguajes para expresarse, valores, metodologías, sus normas, tiempos, para la obtención y verificación de resultados, y formas de organización y control de los recursos.

No es propósito de esta presentación hacer un análisis de otras racionalidades correspondientes a otros "actores sociales", que en un análisis particular de un escenario pudieran realizarse ${ }^{4}$.

La racionalidad política, cuya lógica es la acumulación del poder, con sus códigos, valores de la realidad, mecanismos de control. El decisor político requiere de resultados y

\footnotetext{
3 Rebellato I. La dimensión ética de los procesos educativos, en Cruzando Umbrales. Aportes Uruguayos a la Psicología comunitaria. Luis Jumenes (compilador) Roca Viva. Montevideo.1998. Señala «el neoliberalismo forma parte de un proceso de mundialización llevado adelante por el capitalismo como respuesta a su propia crisis estructural. No sólo es un modelo económico, sino portador de una vision más totalizante articuladora de la dimensión económica, política y cultural. Desde la perspectiva de la cultura tiene un poder de penetración y de conformación que lleva el siguiente mensaje: 1)competividad y exclusión al postular al mercado como el orden natural, la exclusión del sistema es presentada como bajo las exigencias de la intregración mundial capitalista, de la reforma educativa capacitando mano de obra para el nuevo mercado según sus condiciones, el impulso de las políticas sociales de corte compensatorio y los procesos de descentralización que no suponen transformaciones de estructuras injustas, 2) el predominio de la racionalidad instrumental, el valor de la eficiencia y la primacía de lo tecnocrático y 3) la cultura de la desesperanza integrada por la desmovilización, el sometimiento, la frustación y la imposibilidad de una lectura crítica de la realidad.

4 Para el caso de utilizar al escenario como herramienta de diagnóstico, la aplicación de técnicas específicas como el "test de actores sociales" u otros similares permite identificar con precisión la racionalidad de cada actor participante.
} 
que estos sean "visibles" en el momento político adecuado (costo de oportunidad)

Si bien no existe un modelo único de racionalidad política uno de los extremos son estilos de conducción "clientelar", como medio de vinculación con la población, en el otro es un modelo participativo. ${ }^{5}$

La racionalidad técnica con sus teorías y metodologías que fundamentan "encuadres de trabajos", son los fundamentos "científi$\cos ^{\prime \prime}$, que demandan el mayor tiempo posible para la elaboración de diagnósticos y el diseño de las propuestas.

La racionalidad burocrática, con sus tiempos administrativos, con rutinas y estilos aferrados a las normas, poco comprometidos y con resultados que a veces desconoce.

La racionalidad de la población con sus representaciones de la sociedad, de sí mis$\mathrm{ma}$, con sus estrategias de relacionamiento y de obtención de recursos. ${ }^{6}$

Estas racionalidades, a veces son, complementarias, otras fragmentadas o en otros casos enfrentadas entre sí, generando un ámbito complejo, turbulento y con alto grado de incertidumbre que modo de las imágenes de un "caleidoscopio" nos muestran "escenarios" dinámicos y cambiantes para el desempeño del psicólogo comunitario.

\section{Conclusiones}

Se han señalado en este trabajo los componentes del escenario social (necesidades sociales, organización, su relación con el medio ambiente). Se ha contextualizado a los procesos participativos y las relaciones entre actores sociales en un momento histórico de profunda crisis (de representación política frente al poder financiero, de fragmentación social y de modelación de la opinión pública).

Estos elementos constituyen la realidad donde se efectúan las intervenciones comunitarias que, desde la perspectiva de la psicología comunitaria, se orientan a la solución de problemas de la población mediante procesos participativos y que, mediante la reflexión, amplían niveles de concientización y generan nuevas praxis organizativas.

La discusión grupal y comunitaria de cada uno de sus componentes, la contextualización en la realidad inmediata de los grupos o en la situación de la comunidad transforman a la construcción del "escenario" en una herramienta para el diagnóstico, (análisis de problemas, causas y cursos de acción), la planificación participativa y estratégica (relaciones de poder), el diseño de alternativas de acción (procesos decisorios) y las formas organizativas que faciliten la viabilidad de las acciones, potenciando los derechos y deberes de la población y la construcción de articulaciones sociales que faciliten procesos de transformación social.

En el gráfico Nro. 1 - Escenario de la Intervención comunitaria - se describen la totalidad de sus componentes.

5 Para ampliar sobre este tema es interesante el trabajo de Rodrigues, A; Velazquez,F. Municipio y Servicios públicos. Ediciones Sur. Chile. 1994. transparencia y responsabilidad

6 Ej. Las familias de niños desnutridos, que mantienen a uno en tal situación, para poder acceder a los suplementos alimentarios. Las familias que se prestan los hijos o a las embarazadas, para ingresar en las categorías de familia numerosa, con mujer embarazada, para acceder a determinados Programas sociales. 


\section{Gráfico Nro. 1}

\section{Escenario de la Intervención Comunitaria}

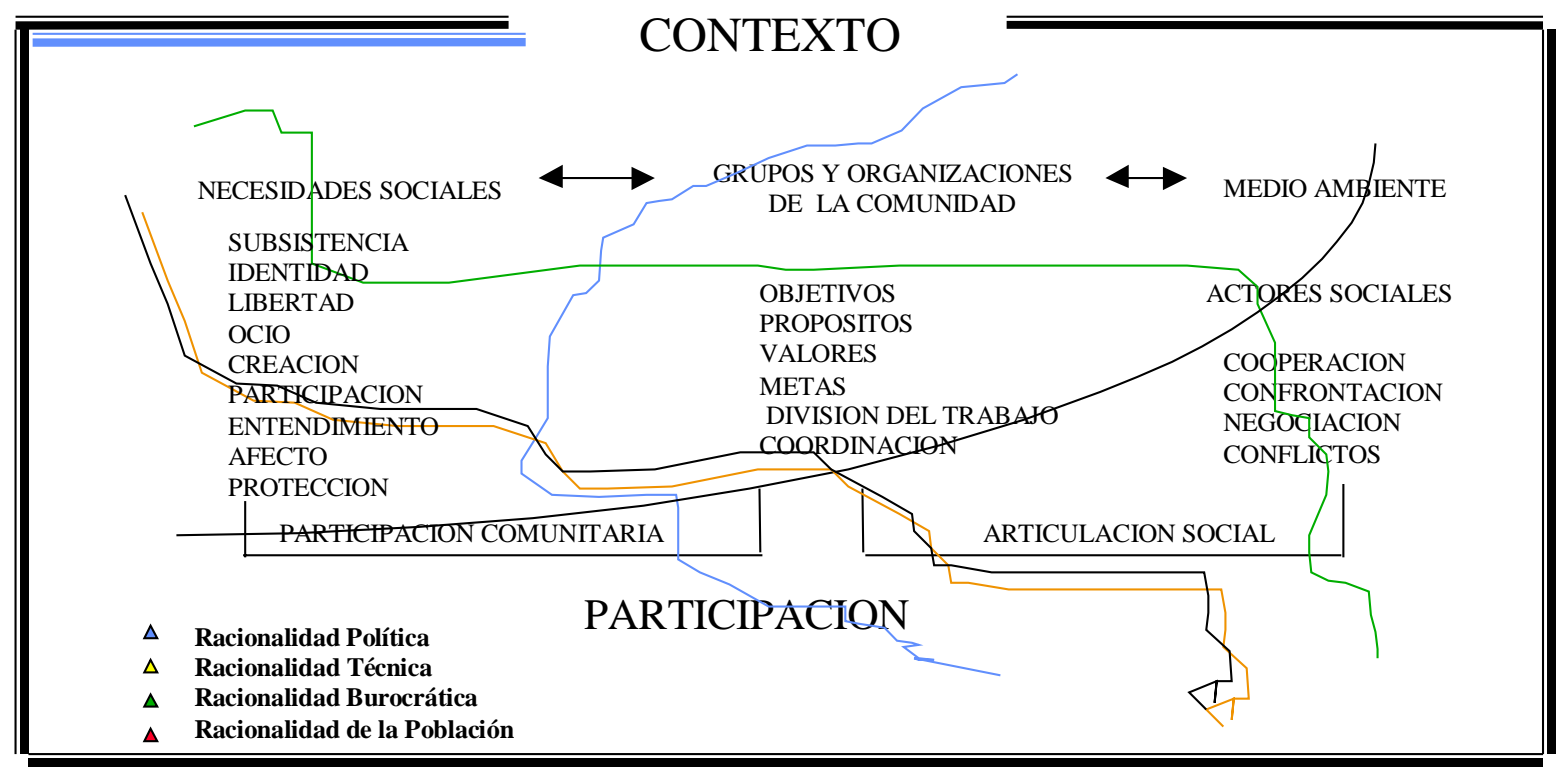

LAPALMA, A.I. 1997

\section{Referencias}

Cardarelli, G; Rosenfeld, M. (1998).Las participaciones de la pobreza. Programas y Proyectos Sociales. Buenos Aires, Paidos.

Chinkes; S; Lapalma, A; Nicesboim,E. (1995) Psicología comunitaria en Argentina. Reconstrución de una práctica psicosocial en la Argentina, en Psicología social comunitaria. Contribuciones Latinoamericanas, Wiesenfeld,E: Sancehes Euclides. Tropykos. Venezuela.

Crozier,M; Friedberg E. (1990) El actor social y el sistema. Las restricciones de la acción colectiva. Alianza Editorial Mexica. Mexico.

Figueroa ,A; Altamirano,T; Sulmont (1996). Exclusión social, desigualdad en el Perú. OIT. Lima.

GADIS-PNUD. (1997) Confines Sociales. Bs-As
Heller, Agnes. (1996).Una Revisión de las teoría de las necesidades. Paidos. Barcelona.

Katz,D., KaHN, R.(1981). Psicología Social de las Organizaciones. Editorial Trillas. .México.

Max-NeEF, M (1993). Desarrollo a escala humana. Nordan-Redes Ediciones. Montevideo.

Montero, M. (1994). Procesos de influencia social conciente e insconciente en el trabajo psicosocial comunitario. La dialéctica entre mayorías y minorías, en Montero M-. Psicología Comunitaria. Universidad de Guadalajara. Guadalajara.

Portes, Alejandro. (1999) Capital Social: Sus orígenes y aplicaciones en la sociología moderna, en De Igual a Igual, Carpio, El desafio del Estado ante los nuevos problemas sociales. J; Novacovsky,I. (compiladores). Fondo de Cultura Económica - FLACSO SIEMPRO. Bs.As 
Rebellato I. (1998). La dimensión ética de los procesos educativos, en Cruzando Umbrales. Aportes Uruguayos a la Psicología comunitaria. Luis Jumenes (compilador) Roca Viva. Montevideo.

SEN,A; (1995) Nuevo examen de la desigualdad. Alianza. Madrid.

SANCHez, Euclides. (1994) Participación Comunitaria y la solución de problemas ambientales en América Latina. Conferencia presentada en el IV Congreso Nacional de Psicología Ambiental. Tenerife. 5,8 de abril.
Vicent, F; Le CleRe, (1998) W. Construcción de la organizaciones ciudadanas de base, en Sostenibilidad de la Sociedad Civil. Estrategias para la movilización de recursos. CIVICUS. Colombia.

Villasante, T (1998). De las redes sociales a las programaciones integrales. Lumen/ Humanitas. Bs.As.

WestergaArd, C; Kelly, J.(1998) Una epistemología contextualista para la investigación ecológica, en Psicología Comunitaria. El enfoque ecológico contextualista de James Kelly.Enrique Saforcada. (Comp). Proa. Bs.As. 